13

\title{
Траекторный анализ в коллекторе с многоступенчатой рекуперацией энергии для прототипа гиротрона DEMO. Часть I. Идеализированное распределение магнитного поля
}

\author{
(C) О.И. Лукша, ${ }^{1}$ П.А. Трофимов, ${ }^{1}$ В.Н. Мануилов, ${ }^{2,3}$ М.Ю. Глявин ${ }^{3}$ \\ ${ }^{1}$ Санкт-Петербургский политехнический университет Петра Великого, \\ 195251 Санкт-Петербург, Россия \\ ${ }^{2}$ Нижегородский государственный университет им. Н.И. Лобачевского, \\ 603950 Нижний Новгород, Россия \\ ${ }^{3}$ Институт прикладной физики РАН, \\ 603950 Нижний Новгород, Россия \\ e-mail: louksha@rphf.spbstu.ru
}

Поступило в Редакцию 9 апреля 2020 г.

В окончательной редакции 9 апреля 2020 г.

Принято к публикации 25 мая 2020 г.

\begin{abstract}
Представлены результаты моделирования коллектора прототипа гиротрона, разрабатываемого для проекта DEMO. Выполнен траекторный анализ в коллекторе с 4-х ступенчатой рекуперацией остаточной энергии пучка, основанной на методе пространственной сепарации электронов в скрещенных азимутальном магнитном и аксиальном электрическом полях. В данной части исследований формирование азимутального магнитного поля осуществлялось с помощью проводника, расположенного на оси прибора. Исследование выполнено для отработанного электронного пучка с распределением частиц по компонентам скорости и координаты, приближенным к полученным в экспериментах с мощными гиротронами. В результате оптимизации геометрии и потенциалов секций коллектора достигнут полный КПД гиротрона более $80 \%$, близкий к максимальному КПД при идеальной сепарации фракций электронного пучка с разной энергией. Полученные данные будут использованы для разработки тороидального соленоида, предназначенного для создания азимутального магнитного поля.
\end{abstract}

Ключевые слова: СВЧ электроника, гиротрон, электронный поток, рекуперация энергии.

DOI: $10.21883 /$ JTF.2021.01.50284.123-20

\section{Введение}

В настоящее время гиротроны являются наиболее мощными источниками СВЧ излучения миллиметрового и субмиллиметрового диапазонов длин волн, способными работать в непрерывном и длинноимпульсном режимах (например, [1,2]). Важнейшей областью применения этих приборов является нагрев плазмы и управление током в установках управляемого термоядерного синтеза (УТС). Разработаны и успешно реализованы гиротронные комплексы для ряда установок УТС на базе токамаков и стеллараторов, включая международный экспериментальный термоядерный реактор ITER. Гиротроны для ITER должны обеспечивать генерацию CВЧ излучения с частотой $170 \mathrm{GHz}$, мощностью $1 \mathrm{MW}$ и полным КПД не менее $50 \%$ в течение 3600 s [3]. Переход к новому поколению термоядерных реакторов предполагает решение ряда физических и инженерных задач, направленных на улучшение параметров, используемых в них гиротронов. Например, для проекта DEMO требуются гиротроны с частотой, превышающей $200 \mathrm{GHz}$, и полным КПД более 60\% [4]. Достижение подобного высокого КПД является одним из основных вызовов, стоящих сегодня перед разработчиками мощных гиротронов.
Повышение полного КПД гиротронов, как и других вакуумных СВЧ приборов, может быть обеспечено за счет рекуперации остаточной энергии винтового электронного потока (ВЭП), не израсходованной при его взаимодействии с высокочастотным полем. Современные мощные гиротроны оснащены системами одноступенчатой рекуперации (single-stage depressed collector), в которых электроны отработанного пучка тормозятся в зазоре между корпусом лампы и изолированным от него коллектором. При одноступенчатой рекуперации КПД гиротрона может быть повышен до 1.5 раз по сравнению с электронным КПД, который в мощных гиротронах, как правило, не превышает 30-35\% [1,2,5]. Следовательно, для достижения полного КПД 60\% и более требуется применение многоступенчатых систем рекуперации, которые предполагают пространственное разделение фракций пучка с разной энергией электронов и их осаждение на секции коллектора под различными потенциалами (например, [6-10]). Трудности практической реализации многоступенчатой рекуперации в гиротронах связаны с наличием относительно сильного магнитного поля в коллекторной области, а также с имеющимся разбросом компонент скорости и радиальных координат ведущих центров электронов в отработанном пучке. Ряд исследований, выполненных в последнее 
время, показал перспективы нового метода сепарации электронов с разной энергией, основанного на их дрейфе в скрещенных электрическом и магнитном полях [1113]. Одной из возможностей является дрейф электронов в азимутальном магнитном и аксиальном электрическом полях. Коллекторная система, в которой используется такой дрейф, была разработана для импульсного гиротрона СПбПУ с рабочей частотой $74.2 \mathrm{GHz}$ и выходной мощностью примерно $100 \mathrm{~kW}$ [14-17]. При комплексном моделировании процессов в этом гиротроне был получен полный КПД, равный $71.2 \%$ за счет как повышения качества ВЭП, так и эффективной многоступенчатой рекуперации [18].

В настоящей работе представлены результаты траекторного анализа коллекторной системы прототипа гиротрона для проекта DEMO. В основе этой системы лежит отработанный в гиротроне СПбПУ метод сепарации электронов, основанный на использовании азимутального магнитного поля. Материал исследований разделен на две части. В первой части рассматривается идеализированное азимутальное магнитное поле, создаваемое расположенным на оси прибора проводником. Во второй части обсуждается коллектор с тороидальным соленоидом, который может быть применен в реальном гиротроне для создания азимутального магнитного поля. Исследование выполнено с использованием программного пакета 3D-моделирования CST Studio Suite [19].

\section{1. Характеристики ВЭП в гиротроне DEMO и особенности расчетной модели}

Текущая версия гиротрона, разработанная в ИПФ РАН, рассчитана на рабочую частоту $250 \mathrm{GHz}$ и ускоряющее напряжение $U_{0}=55 \mathrm{kV}[20,21]$. В центральной плоскости резонатора, где индукция магнитного поля $B_{0}$ равна $9.57 \mathrm{~T}$, основные параметры ВЭП имеют следующие расчетные значения: средний радиус ведущих центров электронных траекторий $R_{0}=3.85 \mathrm{~mm}$, разброс радиусов ведущих центров $\Delta R=0.2 \mathrm{~mm}$.

Важное значение для построения системы рекуперации имеют данные о спектре энергий электронов в отработанном ВЭП. В приведенных ниже расчетах был использован типичный спектр энергии (рис. 1), который был определен на основании экспериментальных данных, полученных в мощных гиротронах [7,22]. Данный спектр соответствовал режиму работы гиротрона с электронным КПД $\eta_{e l}=36 \%$.

При выполнении траекторного анализа плоскостью старта частиц была центральная плоскость резонатора $(z=0)$. В этой плоскости располагались 936 центров эмиссии на радиусах $3.75,3.85,3.95 \mathrm{~mm}$, равномерно распределенные по азимуту. Каждый центр был источником $19 \times 4$ трубок тока. Эти трубки различались энергией частиц и током (всего 19 значений) в соответствии

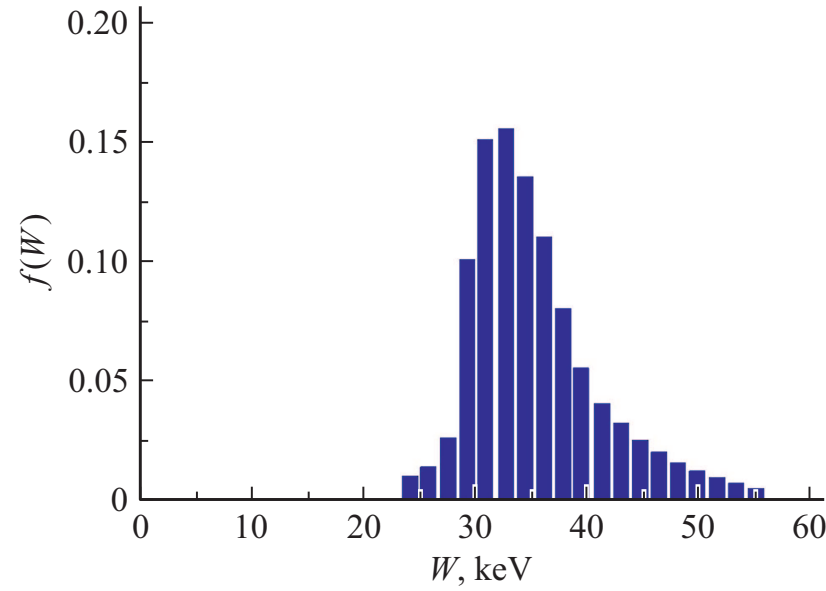

Рис. 1. Распределение электронов по энергиям в отработанном пучке гиротрона.

со спектром, приведенным на рис. 1, и фазой циклотронного вращения (всего 4 значения). Таким образом, суммарно анализировалось 71136 электронных траекторий. При этом для каждой из энергетических фракций было установлено начальное значение питч-фактора, т. е. отношения поперечной скорости к продольной, в диапазоне от 0.1 до 1.2. Для этого использовались данные моделирования взаимодействия ВЭП с высокочастотным полем в гиротроне СПбПУ [18].

Предварительные расчеты электронных траекторий были выполнены с помощью вычислителя Tracking Solver в гиротроне, рассчитанном для работы при одноступенчатой рекуперации. В этом случае коллектор представлял собой цилиндр, на внутренней поверхности которого осаждается электронный пучок. Коллектор соединен конусным переходом с корпусом гиротрона. При движении ВЭП от резонатора до коллектора в спадающем магнитном поле основного соленоида средний радиус пучка увеличивается, и энергия электронов перекачивается из поперечного движения в продольное. При радиусе цилиндрической части коллектора $R_{\text {coll }}=160 \mathrm{~mm}$ область осаждения пучка располагается на расстоянии примерно $z=1270 \mathrm{~mm}$ от центра резонатора. В этой области индукция магнитного поля $B_{\text {coll }}$ равна примерно 0.005 Т. Сильная декомпрессия пучка $\left(B_{0} / B_{\text {coll }} \approx 2000\right)$ обеспечивает приемлемый уровень плотности мощности, рассеиваемой в виде тепла на коллекторе, что принципиально важно при работе гиротрона в непрерывном режиме. При токе пучка $I_{b}=10 \mathrm{~A}$ максимальная плотность мощности $P_{s}$ осаждающегося на коллекторе ВЭП равна примерно $800 \mathrm{~W} / \mathrm{cm}^{2}$ при отсутствии рекуперации.

В случае одноступенчатой рекуперации тормозящий потенциал коллектора задавался равным $-23.6 \mathrm{kV}$ при минимальной энергии электронов в отработанном ВЭП $24.1 \mathrm{keV}$ (рис. 1). В этом режиме отсутствовал ток электронов, отраженных от коллектора вследствие того, что практически вся энергия электронов в области 
торможения сосредоточена в их продольном движении. Мощность $P_{\text {diss }}$, рассеиваемая на коллекторе, равнялась $116 \mathrm{~kW}$ при максимальной плотности мощности $P_{s} \sim 250 \mathrm{~W} / \mathrm{cm}^{2}$. Полный КПД гиротрона при этом составлял $\sim 63 \%$.

\section{2. Модель коллектора с многоступенчатой рекуперацией и распределение полей}

В описанном ниже рекуператоре используется метод пространственной сепарации электронов, основанный на их дрейфе в скрещенных азимутальном магнитном и аксиальном электрическом полях $[12,18,23]$. В первой части работы, представленной настоящей работе, рассматривается идеализированное распределение азимутального магнитного поля, создаваемого с помощью расположенного на оси проводника. В этом случае распределение магнитного поля в коллекторной области однородно по азимуту. Траекторный анализ для такой идеализированной геометрии имеет целью получить данные о возможностях предложенного способа сепарации электронов, которые следует впоследствии учесть при разработке конструкции тороидального соленоида для создания азимутального магнитного поля в реальном приборе. Результаты моделирования коллектора с тороидальным соленоидом будут описаны во второй части работы.

Модель коллекторной области гиротрона показана на рис. 2. Внутренний радиус цилиндрической части корпуса коллектора 1 равен $160 \mathrm{~mm}$. Корректирующие катушки 2 в сочетании с основным соленоидом гиротрона обеспечивают квазиоднородное распределение индукции продольного магнитного поля $B_{z}(z)$ в области $1100<z<1500 \mathrm{~mm}$, где расположены секции коллектора I-IV. На рис. 3 показано распределение $B_{z}(z)$ на радиусе, примерно равном среднему радиусу $R_{b}$ невозмущенного ВЭП при его движении в отсутствие напряжения на секциях коллектора. Азимутальное магнитное поле создается с помощью проводника, зада-

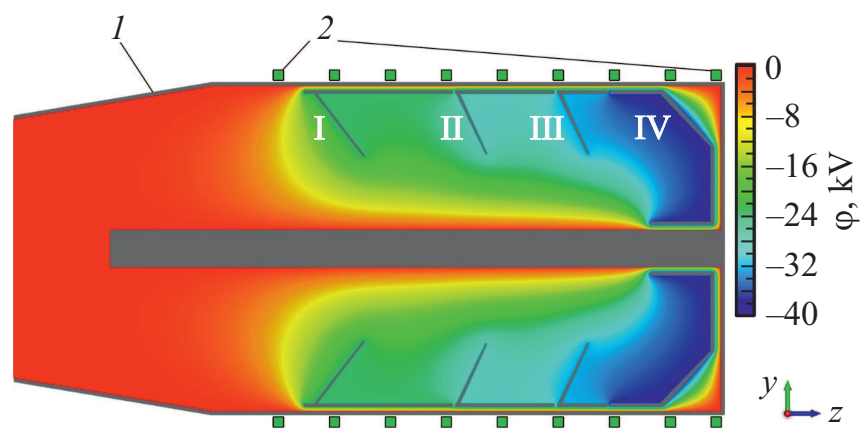

Рис. 2. Схематическое изображение модели коллектора и распределение потенциала $\varphi$ в плоскости $y-z .1$ - корпус коллектора, 2 - корректирующие катушки, I-IV - секции коллектора.

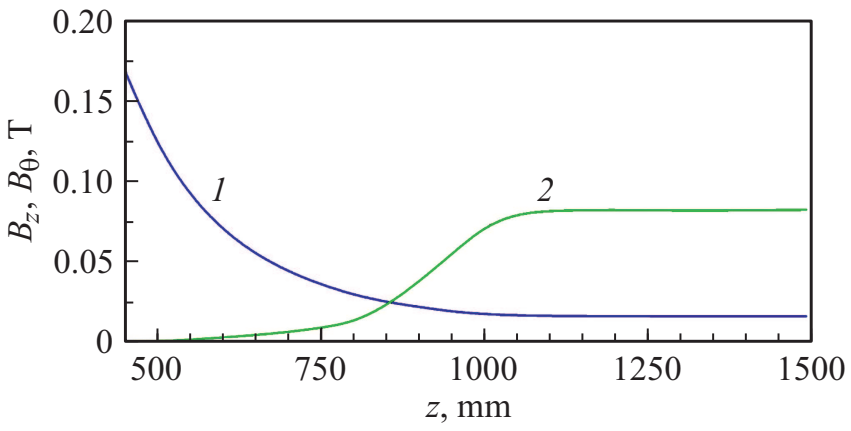

Рис. 3. Зависимости аксиальной $B_{z}$ (кривая 1 ) и азимутальной $B_{\theta}$ (кривая 2) компонент индукции магнитного поля, определенных при $r \approx R_{b}$, от продольной координаты $z$.

ваемого как источник Coil Segment Source в вычислителе Magnetostatic Solver. Выбором геометрии проводника обеспечивается адиабатичность распределения азимутального магнитного поля в переходной области между резонатором и коллектором. Соответствующая зависимость $B_{\theta}(z)$ при $r \approx R_{b}$ также показана на рис. 3 .

Электрическое поле создается с помощью четырех секций-электродов конусной формы, потенциал которых уменышается по мере удаления от резонатора (рис. 2). На оси прибора располагается соединенная c корпусом труба, внутри которой будут находиться витки внутренней обмотки тороидального соленоида. Были выбраны следующие значения потенциалов секций $U_{\mathrm{I}}=-23.6 \mathrm{kV}, U_{\mathrm{II}}=-29.1 \mathrm{kV}, U_{\mathrm{III}}=-32.4 \mathrm{kV}$, $U_{\mathrm{IV}}=-37.8 \mathrm{kV}$ (потенциалы корпуса прибора и коллектора равны нулю). При этих значениях $U_{\mathrm{I}}-U_{\mathrm{IV}}$ достигает максимума полный КПД гиротрона в случае идеальной сепарации, когда каждая из энергетических фракций ВЭП осаждается на соответствующей секции коллектора. В настоящей работе решалась многопараметрическая задача по достижению минимальной мощности $P_{\text {diss }}$ за счет выбора оптимальной геометрии секций коллектора. Значение $P_{\text {diss }}$ определялось в результате траекторного анализа, описанного в следующем разделе. Распределение потенциала в области торможения электронов при оптимальной геометрии секций и указанных выше значениях потенциалов $U_{\mathrm{I}}-U_{\mathrm{IV}}$ показано на рис. 2.

В процессе оптимизации геометрии коллектора принималась во внимание необходимость обеспечения малой величины тока электронов, отраженных от него в сторону резонатора. Этот фактор также учитывался при выборе значений $U_{\mathrm{I}}-U_{\mathrm{IV}}$. Отражение электронов от коллекторной области ограничивает возможности повышения тормозящего напряжения при работе электронных вакуумных приборов с рекуперацией. В гиротронах отраженные частицы, двигаясь вдоль силовых линий магнитного поля, могут достигать резонатора и негативно влиять на эффективность преобразования энергии электронного пучка в энергию СВЧ поля [24-27]. Как показывают проведенные ранее иссле- 
дования, предельный уровень отражения от коллектора, при превышении которого падает выходная мощность, в различных гиротронах может различаться и достигать единиц процентов [28]. Преимуществом описываемого в настоящей работе рекуператора является возможность перехвата отраженных, а также вторичных электронов секциями коллектора и его корпусом на этапе движения в скрещенных $E \times B$ полях, когда частицы смещаются в радиальном направлении $[12,18]$.

\section{3. Результаты траекторного анализа коллектора с четырехступенчатой рекуперацией}

Были исследованы два режима работы коллектора при различных значениях индукции азимутального магнитного поля $B_{\theta}$ в области торможения ВЭП $(z>1100 \mathrm{~mm})$, равных $\sim 0.08$ и 0.13 Т. Двигаясь в этой области, электроны делают несколько оборотов вокруг оси $z$. Число оборотов зависит, очевидно, от отношения $B_{\theta} / B_{z}$ и от времени до осаждения электронов на поверхности какого-либо электрода. На рис. 4 показано положение частиц в плоскости $x=0$ при $B_{\theta}=0.08 \mathrm{~T}$, полученное как результат пересечения винтообразных электронных траекторий с этой плоскостью. Рис. 5 демонстрирует проекции отдельных траекторий на плоскость $r-z$ для различной начальной энергии электронов. Из рис. 4 и 5 видно, что по мере движения частиц в тормозящем электрическом поле происходит уменьшение их энергии и смещение на более высокие радиусы под действием скрещенных $E \times B$ полей. При изменении начальной энергии изменяется вид траекторий - с ростом энергии электроны проходят бо́льшее расстояние вдоль оси $z$ и осаждаются на секции под более отрицательным потенциалом.

Важно, что основная часть частиц осаждается на задней поверхности секций после изменения направления продольной скорости (см. также рис. 6). Как результат, возрастает время, в течение которого электроны дрейфуют в радиальном направлении. Это приводит к увеличению площади осаждения электронного пучка

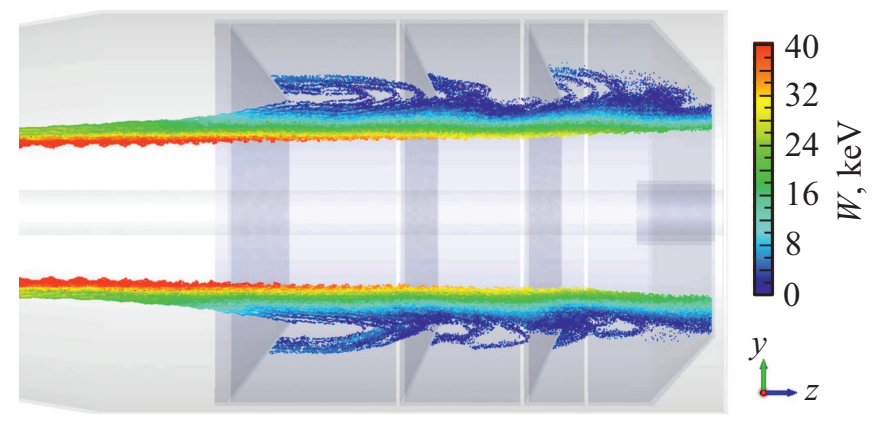

Рис. 4. Положение частиц в плоскости $x=0$ (цвет соответствует энергии частиц $W$ ).

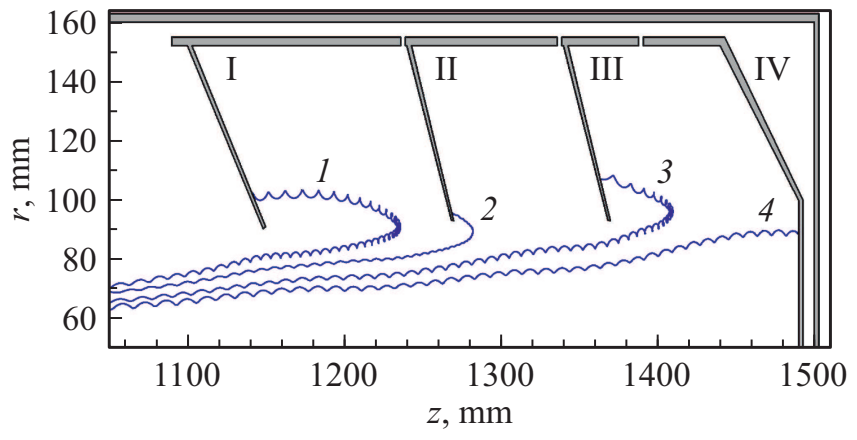

Рис. 5. Траектории электронов в плоскости $r-z$. Значения начальной энергии $W: 1-25.7,2-29.2,3-36.1$, $4-41.3 \mathrm{keV}$.

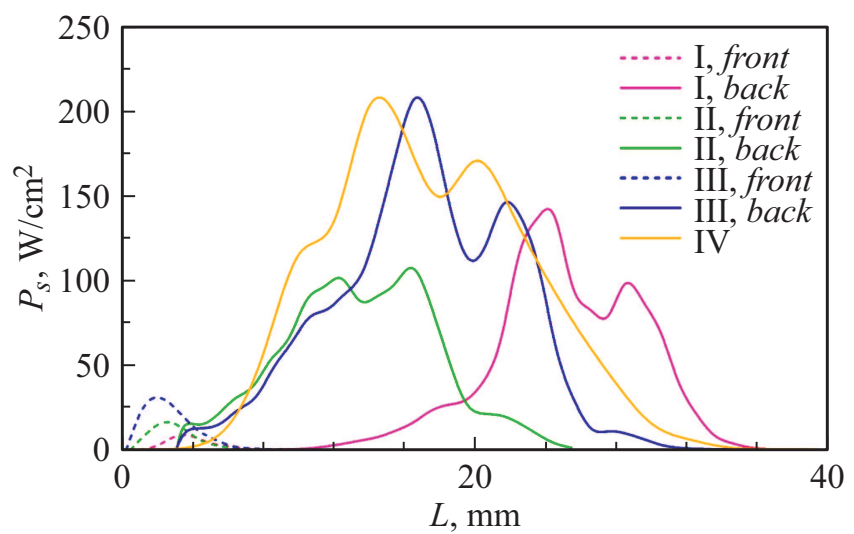

Рис. 6. Распределения плотности мощности $P_{s}$ осаждающегося ВЭП вдоль образующей $L$ конусных секций коллектора I-IV c усреднением по азимутальной координате. Для секций I-III распределения приведены отдельно для передней (front) и задней (back) их поверхностей. Для секции IV направление вдоль $L$ совпадает с радиальным направлением.

Результаты траекторного анализа рекуператора гиротрона

\begin{tabular}{c|c|c}
\hline Параметр & $B_{\theta}=0.08 \mathrm{~T}$ & $B_{\theta}=0.13 \mathrm{~T}$ \\
\hline$P_{\mathrm{I}}, \mathrm{kW}$ & 6.87 & 6.78 \\
$P_{\mathrm{II}}, \mathrm{kW}$ & 5.52 & 7.84 \\
$P_{\mathrm{III}}, \mathrm{kW}$ & 11.02 & 11.38 \\
$P_{\mathrm{IV}}, \mathrm{kW}$ & 13.58 & 13.51 \\
$P_{\text {diss }}, \mathrm{kW}$ & 36.99 & 39.51 \\
$I_{\text {ref }}, \mathrm{kW}$ & $1.2 \cdot 10^{-4} I_{b}$ & $1.1 \cdot 10^{-3} I_{b}$ \\
$\eta_{t}, \%$ & 84.3 & 83.3
\end{tabular}

и положительно сказывается на тепловой нагрузке на секции коллектора.

Основные данные, характеризующие работу рекуператора при $B_{\theta}=0.08$ и $0.13 \mathrm{~T}$, сведены в таблицу. Здесь приведены значения мощности, рассеиваемой на каждой из секций $P_{\mathrm{I}}-P_{\mathrm{IV}}$ и суммарно на всем коллекторе $P_{d i s s}$, тока электронов $I_{r e f}$, отраженных от коллектора и проходящих в резонатор, а также полного КПД 
гиротрона $\eta_{t}$, рассчитанного по формуле:

$$
\eta_{t}=\frac{P_{\mathrm{RF}}}{P_{\mathrm{RF}}+P_{d i s s}}
$$

где $P_{\mathrm{RF}}=\eta_{e l} I_{b} U_{0}=198 \mathrm{~kW}$ - выходная СВЧ мощность. Достигнутый полный КПД довольно высок и близок к максимальному возможному значению этой величины для четырехступенчатой схемы рекуперации. Некоторое снижение КПД следует ожидать, если в расчетах мы будем использовать отработанный пучок с распределением частиц по энергиям и координатам, в боо́льшей степени приближенным к реальным условиям (см., например, [18]).

В рассчитанных режимах поток электронов, отраженных от коллектора, мал и не должен заметно влиять на электронный КПД гиротрона. Малое отражение в первую очередь связано с уменьшенным значением напряжения $\left|U_{\mathrm{I}}\right|$. За счет повышения тормозящего напряжения на первой секции, в принципе, можно добиться дополнительного увеличения полного КПД даже в присутствии заметного отражения электронов от коллектора, когда уменьшены выходная СВЧ мощность и электронный КПД [27].

Распределение плотности мощности $P_{s}$, выделяемой на секциях коллектора, показано на рис 6. Для секций I-III расстояние $L$ отсчитывается от края (основания усеченного конуса) каждой из секций вдоль ее образующей отдельно для внутренней и наружной поверхностей. Для секции IV направление вдоль $L$ совпадает с радиальным направлением $(L=0$ соответствует $r=60 \mathrm{~mm})$. Для получения этих распределений было выполнено усреднение, при котором площадь осаждения каждой из электронных траекторий (трубок тока) принималась приблизительно равной квадрату расстояния между соседними траекториями. Каждая из секций испытывает определенную тепловую нагрузку - значения мощности $P_{\mathrm{I}}-P_{\mathrm{IV}}$ отличаются друг от друга не более, чем в три раза. При этом максимальная плотности мощности близка к значению этой величины для одноступенчатой рекуперации. Очевидно, что дополнительного снижения плотности мощности для рассматриваемой конструкции четырехступенчатого рекуператора можно добиться за счет снижения индукций магнитного поля $B_{z}$ и $B_{\theta}$, что приведет к увеличению радиусов ведущих центров электронных орбит в области рекуперации, а также за счет изменения углов наклона поверхностей секций, на которые осаждаются электроны.

\section{Заключение}

Таким образом, выполненное в работе моделирование коллектора для гиротрона, который был разработан как прототип для гиротрона DEMO, подтвердило возможности эффективной сепарации электронов отработанного ВЭП, основанной на их дрейфе в скрещенных азимутальном магнитном и аксиальном электрическом полях.
Разработанная конструкция 4-х ступенчатого рекуператора при идеализированном азимутальном магнитном поле, создаваемом приосевым проводником, обеспечивает достижение полного КПД гиротрона, превышающего $80 \%$. Полученные данные будут использованы при проектировании тороидального соленоида, который может быть использован для создания азимутального магнитного поля в реальном приборе.

\section{Финансирование работы}

Исследование выполнено за счет гранта Российского научного фонда (проект № 16-12-10010). Часть результатов была получена с использованием вычислительных ресурсов суперкомпьютерного центра СанктПетербургского политехнического университета Петра Великого (http://www.scc.spbstu.ru). Разработка прототипа гиротрона для DEMO ведется в рамках проекта Российского научного фонда 19-79-30071 и все требования к ЭОС сформулированы в рамках этого гранта.

\section{Конфликт интересов}

Авторы заявляют, что у них нет конфликта интересов.

\section{Список литературы}

[1] A.G. Litvak, G.G. Denisov, V.E. Myasnikov, E.M. Tai, E.A. Azizov, V.I. Ilin. J. Infrared Millimeter Terahertz Waves, 32 (3), 337 (2011).

[2] M. Thumm. J. Infrared Millimeter Terahertz Waves, 41 (1), 1 (2020).

[3] C. Darbos et al. J. Infrared Millimeter Terahertz Waves, 37 (1), 4 (2016).

[4] J. Jelonnek et al. Fusion Engineering and Design, 123, 241 (2017).

[5] V.N. Manuilov, M.V. Morozkin, O.I. Luksha, M.Y. Glyavin. Infrared Physics Technol., 91, 46 (2018).

[6] H.G. Kosmahl. Proc. IEEE, 70 (11), 1325 (1982).

[7] A.L. Goldenberg, V.N. Manuilov, M.A. Moiseev, N.A. Zavolsky. Int. J. Infrared Millimeter Waves, 18(1), 43 (1996).

[8] A. Singh, S. Rajapatirana, Y. Men, V.L. Granatstein, R.L. Ives, A.J. Antolak. IEEE Trans. Plasma Sci., 27 (2), 490 (1999).

[9] G. Ling, B. Piosczyk, M.K. Thumm. IEEE Trans. Plasma Sci., 28 (3), 606 (2000).

[10] М.Ю. Глявин, М.В. Морозкин, М.И. Петелин. Известия вузов. Радиофизика, 49 (10), 900 (2006). [M.Y. Glyavin, M.V. Morozkin, M.I. Petelin. Radiophys. Quantum Electron., 49 (10), 811 (2006).]

[11] I.Gr. Pagonakis, J.-P. Hogge, S. Alberti, K.A. Avramides, J.L. Vomvoridis. IEEE Trans. Plasma Sci., 36 (2), 469 (2008).

[12] О.И. Лукша, П.А. Трофимов. Письма в ЖТФ, 41 (18), 38 (2015). [O.I. Louksha, P.A. Trofimov. Tech. Phys. Lett., 41 (9), 884 (2015).]

[13] C. Wu, I.G. Pagonakis, K.A. Avramidis, G. Gantenbein, S. Illy, M. Thumm M., J. Jelonnek. Phys. Plasmas, 25 (3), 033108 (2018). 
[14] O. Louksha, B. Piosczyk, G. Sominski, M. Thumm, D. Samsonov. IEEE Trans. Plasma Sci., 34 (3), 502 (2006).

[15] Д.В. Касьяненко, О.И. Лукша, Б. Пиосчик, Г.Г. Соминский, М. Тумм. Известия вузов. Радиофизика, 47 (5-6), 463 (2004). [D.V. Kas'yanenko, O.I. Louksha, B. Piosczyk, G.G. Sominsky, M. Thumm. Radiophys. Quantum Electron., $47(5-6), 414$ (2004).

[16] О.И. Лукша, Д.Б. Самсонов Г.Г. Соминский, А.А. Цапов. ЖТФ, 82 (6), 101 (2012). [O.I. Louksha, D.B. Samsonov, G.G. Sominskii, A.A. Tsapov. Tech. Phys., 57 (6), 835 (2012).]

[17] Д.В. Борзенков, О.И. Лукша. ЖТФ, 67 (9), 98 (1997). [D.V. Borzenkov, O.I. Luksha. Tech. Phys., 42(9), 1071 (1997).]

[18] О.И. Лукша, П.А. Трофимов. ЖТФ, 89 (12), 1988 (2019). [O.I. Louksha, P.A. Trofimov. Tech. Phys., 64 (12), 1889 (2019).]

[19] Электронный ресурс. Режим доступа: https://www.3ds.com/products-services/simulia/products/cststudio-suite/

[20] M. Glyavin, V. Manuilov, M. Morozkin. Proc. 43rd Int. Conf. Infrared, Millimeter and Terahertz Waves. (Nagoya, Japan, 2018). 8510139.

[21] G.G. Denisov et al. Rev. Sci. Instrum., 89 (8), 084702 (2018),

[22] Н.П. Венедиктов, М.Ю. Глявин, А.Л. Гольденберг, В.Е. Запевалов, А.Н. Куфтин, М.А. Моисеев, А.С. Постникова. ЖТФ, 89 (12), 63 (2000). [N.P. Venediktov, M.Yu. Glyavin, A.L. Goldenberg, V.E. Zapevalov, A.N. Kuftin, M.A. Moiseev, A.S. Postnikova. Tech. Phys., 45 (12), 1571 (2000).]

[23] O.I. Louksha, P.A. Trofimov. Proc. 18th Int. Vacuum Electronics Conf., IVEC 2017 (London, United Kingdom, 2017) p. 1.

[24] K. Sakamoto, M. Tsuneoka, A. Kasugai, T. Imai, T. Kariya, K. Hayashi, Y. Mitsunaka. Phys. Rev. Lett., 73 (26), 3532 (1994).

[25] Н.П. Венедиктов, М.Ю. Глявин, В.Е. Запевалов, А.Н. Куфтин. Известия вузов. Радиофизика, 41 (5), 670 (1998). [N.P. Venediktov, M.Yu. Glyavin, V.E. Zapevalov, A.N. Kuftin. Radiophys. Quantum Electron. 41 (5), 449 (1998).]

[26] M.V. Morozkin, M.Y. Glyavin, G.G. Denisov, A.G. Luchinin. Int. J. Infrared Millimeter Waves, 29 (11), 1004 (2008).

[27] Н.А. Завольский, В.Е. Запевалов, А.Н. Куфтин, А.С. Постникова. Мат. 28-й Междунар. Крымской конф. „СВЧтехника и телекоммуникационные технологии“ (Севастополь, 2018) с. 1131.

[28] B. Piosczyk, C.T. Iatrou, G. Dammertz, M. Thumm. IEEE Trans. Plasma Sci., 24 (3), 579 (1996). 